\title{
Identifying the Role of Novel Protein Kinase C Isoforms in Mediating Paclitaxel-Induced Peripheral Neuropathy
}

\author{
Amanda L. Blaker, Carmen M. Mitchell, and 타in A. Semple \\ Department of Neurosciences and Neurological Disorders, Biomedical Sciences Graduate Program, University of Toledo College of Medicine, Toledo, Ohio \\ 43614 \\ Review of He and Wang
}

Paclitaxel (Taxol) is a commonly prescribed chemotherapeutic drug that stabilizes microtubules and consequently prevents progression of mitosis in rapidly dividing tumor cells. Like many other chemotherapeutics, paclitaxel is not selective, and it can therefore produce many undesired side effects. One debilitating effect experienced by individuals receiving the drug is paclitaxelinduced peripheral neuropathy (PIPN). The neuropathy is characterized by spontaneous pain, as well as evoked hyperalgesia and allodynia. Due to a limited physiological understanding of PIPN, there are few treatments available for patients experiencing PIPN (Scripture et al., 2006).

Multiple second messenger signaling molecules have been implicated in the pathophysiology of PIPN, including cAMP, protein kinase $\mathrm{A}$, and protein kinase $\mathrm{C}$ (PKC) (Hucho and Levine, 2007). Although each is necessary for pain transmission, $\mathrm{PKC}$ plays a predominant role in the nociceptive pathway involved in chronic pain and PIPN. Specifically, inhibition of the PKC $\varepsilon$ isoform in chronic pain models has been shown to block hyperalgesia, whereas inhibition of other second messengers, like

Received May 17, 2015; revised June 17, 2015; accepted June 22, 2015.

The authors gratefully acknowledge Dr. Marthe Howard and Dr. Joseph Margiotta, co-course directors of the Neurosciences and Neurological Disorders Advanced Journal Club course at University of Toledo College of Medicine, for their mentorship throughout the course.

Correspondence should be addressed to Amanda L. Blaker, Department of Neurosciences, University of Toledo College of Medicine, 3000 Arlington Avenue, Toledo, OH 43614. E-mail: amanda.blaker@rockets.utoledo.edu. DOI:10.1523/JNEUROSCI.1903-15.2015

Copyright $\odot 2015$ the authors $\quad 0270-6474 / 15 / 3510101-02 \$ 15.00 / 0$
cAMP, only partially block hyperalgesia. Many chronic pain studies have focused on the role of PKC in evoked pain, while neglecting its role in spontaneous pain. Furthermore, in attempts to understand chronic pain, most research has focused primarily on $\mathrm{PKC} \varepsilon$; other isoforms of $\mathrm{PKC}$ (e.g., PKC $\beta I I$ and PKC $\delta$ ) have yet to be tested. In a recently article published in The Journal of Neuroscience, He and Wang (2015) set out to further investigate the unique roles of various PKC isoforms in both spontaneous and evoked adverse pain associated with rodent models of PIPN.

Because the dorsal root ganglion (DRG) is an important site for excitatory nociceptive transmission, He and Wang (2015) focused their investigation in this region. Upon in vitro application of paclitaxel, $\mathrm{PKC} \beta \mathrm{II}$ and $\mathrm{PKC} \delta$, in addition to $\mathrm{PKC} \varepsilon$, translocated to the plasma membrane that indicated an activated state. To determine the importance of PKC isoform activation in PIPN, He and Wang (2015) pharmacologically manipulated PKC by using specific PKC isoform inhibitors to block paclitaxel's effects on isolated DRG neurons. In normal pain transmission, calcitonin gene-related peptide (CGRP), an excitatory neuropeptide, is released from primary sensory afferents. CGRP was upregulated with paclitaxel exposure, and inhibiting any of the three PKC isoforms restored CGRP to basal concentrations, indicating that these $\mathrm{PKC}$ isoforms were involved in CGRP regulation. These results confirmed the previously established role for PKC $\varepsilon$ in pain transmis- sion, and identified a novel role for PKC $\beta \mathrm{II}$ and $\mathrm{PKC} \delta$ as two additional PKC isoforms activated by paclitaxel treatment that are necessary for CGRP release in the nociceptive pathway and thus possibly in pain transmission.

The activation of multiple $\mathrm{PKC}$ isoforms after paclitaxel exposure raises the possibility that differential isoform activation is associated with functional differences in pain transmission. Pain transmission can be influenced by differential expression of specific molecules on the plasma membrane of nociceptors. Generally, DRG nociceptors can be divided into two categories depending on whether the cell binds isolectin $\mathrm{B}_{4}$ $\left(\mathrm{IB}_{4}\right)$. Both $\mathrm{IB}_{4}$-negative and $\mathrm{IB}_{4}$-positive nociceptors are involved in pain transmission, but much speculation has been made regarding functional differences such as action potential threshold and duration, and response to different types of stimuli (Snider and McMahon, 1998; Stucky and Lewin, 1999). He and Wang (2015) revealed that paclitaxel treatment activated all three PKC isoforms in both $\mathrm{IB}_{4}$-positive and $\mathrm{IB}_{4}$ negative afferents, but PKC $\beta$ II was more prominently activated in $\mathrm{IB}_{4}$-negative neurons than in $\mathrm{IB}_{4}$-positive neurons. Unlike $\mathrm{IB}_{4}$-positive nociceptors, $\mathrm{IB}_{4}$-negative nociceptors contain CGRP. This fits nicely with data showing that inhibition of PKC $\beta$ II was most effective at blocking CGRP release. Based on these results, it is reasonable to speculate that PKC $\beta$ II may have a different role than other isoforms in mediating pain 
transmission due to its prominence in $\mathrm{IB}_{4}$ negative nociceptors.

To confirm their in vitro findings and further investigate the roles of PKC isoforms, He and Wang (2015) used a previously established in vivo mouse model of PIPN, which develops hyperalgesia to mechanical and thermal stimuli as seen in humans (Chen et al., 2011). Consistent with previous studies, PIPN mice were hypersensitive to mechanical and thermal stimuli $20 \mathrm{~d}$ after intrathecal paclitaxel administration. Adding PKC $\varepsilon$, PKC $\beta \mathrm{II}$, or PKC $\delta$ isoform antagonists raised the evoked pain threshold to that of controls. This suggests that all three PKC isoforms play a critical role in the hypersensitivity to evoked pain experienced after paclitaxel administration.

Until recently, a major limitation in this field of research has been our inability to measure spontaneous pain in animal models, which is an important aspect of PIPN in humans. In 2012, He et al. established a conditioned place preference (CPP) behavioral paradigm to investigate spontaneous pain in mice following nerve injury. In this model, lidocaine and saline are each paired with a respective chamber. PIPN mice preferred the chamber where they received lidocaine, suggestive of pain reduction by the non-rewarding lidocaine injection. After replicating these results in PIPN mice, He and Wang (2015) explored the role of PKC isoforms in spontaneous pain by replacing the lidocaine injections with PKC inhibitors. PKC $\beta \mathrm{II}$ and $\mathrm{PKC} \delta$ inhibitors induced $\mathrm{CPP}$ in a manner similar to the lidocaine preference, indicative of spontaneous pain inhibition, but, interestingly, $\mathrm{PKC} \varepsilon$ did not. $\mathrm{PKC} \beta \mathrm{II}$ inhibition also had a greater effect than $\mathrm{PKC} \delta$ inhibition on spontaneous pain blockade. Given that PKC $\beta$ II activation corresponded most strongly with $\mathrm{IB}_{4}{ }^{-}$ negative cells, this provides evidence that evoked and spontaneous pain might be transmitted through partially non-overlapping pathways. Both IB4negative cells and CGRP release have been established in the literature as indicators of pain transmission (Stucky and Lewin, 1999). Because the results described by He and Wang (2015) show a strong association between PKC $\beta$ II and IB4-negative cells, and between PKC $\beta$ II and CGRP release, this strengthens the growing evidence of PKC $\beta I I$ involvement in nociceptive signaling pathways. Thus, these findings not only implicate two additional PKC isoforms in experiencing pain, but contribute to our understanding of how evoked and spontaneous pain may differ mechanistically, namely through $\mathrm{PKC} \varepsilon$.

Another characteristic of nociceptors is the presence of vanilloid receptor 1 (TRPV1), a downstream molecule that could be differentially affected by different PKC isoforms. Colabeling for the specific PKC isoforms and TRPV1 demonstrated expression of TRPV1 on nociceptors expressing all three PKC isoforms (He and Wang, 2015). These receptors are essential for hyperalgesia to particular stimuli, and they have been implicated in various neuropathies (Holzer, 2008). Mechanistically, PKC $\varepsilon$ is known to increase TRPV1 membrane insertion and increase TRPV-dependent sodium influx via phosphorylation (Velázquez et al., 2007). Phosphorylative changes lead to increased neuronal firing in primary afferents that project to the DRG, resulting in hypersensitivity to non-noxious stimuli. With the established role of TRPV 1 in hypersensitive responses after PKC $\varepsilon$ activation, it would be valuable to investigate whether the other two isoforms identified in this paper, $\mathrm{PKC} \beta \mathrm{II}$ and $\mathrm{PKC} \delta$, affect TRPV1 phosphorylation and subsequent sodium influx. Furthermore, studies examining how the degree of phosphorylation of TRPV1 relates to pain threshold in vivo could provide more insight to downstream mechanisms of PKC activation in peripheral neuropathies, such as PIPN.

Knowledge of specific PKC isoform activation in spontaneous and evoked pain may aid in the development of clinical treatments. Nonspecific PKC inhibitors have been tested with little success in the treatment of a variety of chronic-painrelated conditions due to their lack of selectivity and limitations of their pharmacokinetics (Mochly-Rosen et al., 2012). Targeting a specific isoform, as $\mathrm{He}$ and Wang (2015) did, could potentially reduce the risk of side effects. Interestingly, targeting specific PKC isoforms, such as $\mathrm{PKC} \beta$, has shown promise in managing diabetic neuropathy (MochlyRosen et al., 2012). This evidence further supports the involvement of PKC $\beta$ II in both spontaneous and evoked pain and provides rationale for therapeutically targeting this isoform in aversive neuropathies. An additional factor that may affect the specificity of a treatment is how it is administered. Intrathecal administration, as used by $\mathrm{He}$ and Wang (2015), is a method to selectively target the DRG. Nonsystemic routes of administration, including intrathecal, could be considered in the clinical treatment of pain to reduce off-target effects.
In summary, these results contribute significantly to our understanding of the mechanisms underlying PIPN. He and Wang (2015) provide compelling evidence for the involvement of three PKC isoforms in PIPN, two of which have been previously neglected in pain research. Importantly, the greatest effects on pain reduction were seen by blocking PKC $\beta I I$ activation. Another major implication of these experiments is the dichotomy of evoked and spontaneous pain. This study provides novel evidence for differential $\mathrm{PKC}$ involvement in spontaneous versus evoked pain. Namely, PKC $\beta \mathrm{II}$ and $\mathrm{PKC} \delta$, but not $\mathrm{PKC} \varepsilon$, were shown to play a role in spontaneous pain. Since there are many aspects of pain that are poorly understood, this information could potentially change how researchers investigate pain signaling. The exciting results from these studies open new avenues for expanding our understanding of pain and improving current treatments for patients with PIPN.

\section{References}

Chen Y, Yang C, Wang ZJ (2011) Proteinaseactivated receptor 2 sensitizes transient receptor potential vanilloid 1 , transient receptor potential vanilloid 4 , and transient receptor potential ankyrin 1 in paclitaxel-induced neuropathic pain. Neuroscience 193:440-451. CrossRef Medline

He Y, Tian X, Hu X, Porreca F, Wang ZJ (2012) Negative reinforcement reveals non-evoked ongoing pain in mice with tissue or nerve injury. J Pain 13:598-607. CrossRef Medline

He Y, Wang ZJ (2015) Nociceptor beta II, delta, and epsilon isoforms of PKC differentially mediate paclitaxel-induced spontaneous and evoked pain. J Neurosci 35:4614-4625. CrossRef Medline

Holzer P (2008) The pharmacological challenge to tame the transient receptor potential vanilloid-1 (TRPV1) nocisensor. Br J Pharmacol 155:1145-1162. CrossRef Medline

Hucho T, Levine JD (2007) Signaling pathways in sensitization: toward a nociceptor cell biology. Neuron 55:365-376. CrossRef Medline

Mochly-Rosen D, Das K, Grimes KV (2012) Protein kinase $C$, an elusive therapeutic target? Nat Rev Drug Discov 11:937-957. CrossRef Medline

Scripture CD, Figg WD, Sparreboom A (2006) Peripheral neuropathy induced by paclitaxel: recent insights and future perspectives. Curr Neuropharmacol 4:165-172. CrossRef Medline

Snider WD, McMahon SB (1998) Tackling pain at the source: new ideas about nociceptors. Neuron 20:629-632. CrossRef Medline

Stucky CL, Lewin GR (1999) Isolectin B4positive and-negative nociceptors are functionally distinct. J Neurosci 19:6497-6505. Medline

Velázquez KT, Mohammad $\mathrm{H}$, Sweitzer SM (2007) Protein kinase C in pain: involvement of multiple isoforms. Pharmacol Res 55:578589. CrossRef Medline 